\title{
What I see is what I want: Top-down attention biasing choice behavior ${ }^{\text {th }}$
}

\author{
M. Vriens ${ }^{\mathrm{a}, *}$, C. Vidden ${ }^{\mathrm{b}}$, J. Schomaker ${ }^{\mathrm{c}}$ \\ ${ }^{a}$ Department of Marketing, University of Wisconsin, La Crosse, USA \\ ${ }^{\mathrm{b}}$ Department of Mathematics, University of Wisconsin, La Crosse, USA \\ ${ }^{\mathrm{c}}$ Faculty of Social and Behavioural Sciences, Section Health, Medical \& Neuropsychology, Leiden University, the Netherlands
}

\section{A R T I C L E I N F O}

\section{Keywords:}

Decision-making

Attention

Value-based choices

Eye tracking

Buyer choices

Marketing

\begin{abstract}
A B S T R A C T
When making a purchasing decision, people typically scan the available options before deciding. Previous studies have shown that bottom-up stimulus-driven factors can influence choice behavior through their effect on attention, but studies that investigate the effect of top-down attention are scarce. Here, we investigate the role of top-down attention, i.e. by using directional and neutral cues, in a choice task using eye fixations as a proxy of attention. On each trial, participants chose a preferred food item amongst two similarly valued options. Attention was manipulated using directional and neutral cues. We show that directional cues have a significant effect on attention, and attention has a significant effect on choice. A mediation analysis confirms the indirect effect of cues on choice. This suggests that cues can be used to guide attention to consumer products and affect purchasing decisions.
\end{abstract}

\section{Introduction}

Attention as a concept has become increasingly important in the study of consumer behavior. Eye movements and fixations are typically used as measures of attention (e.g. Deubel \& Schneider, 1996 and Orquin \& Mueller Loose, 2013), and a range of studies show that attention and decision-making are tightly linked (e.g. Krajbich, Armel, \& Rangel, 2010; Orquin \& Mueller Loose, 2013; Peschel, Orquin, \& Mueller Loose, 2019; Pieters, Warlop, \& Wedel, 2002; Schotter, Berry, McKenzie, \& Rayner, 2010; Towal, Mormann, \& Koch, 2013). Consumers often scan the available options prior to making a choice; for example, when scanning products on a supermarket shelf (e.g. Gidlöf, Anikin, Lingonblad, \& Wallin, 2017). Eye movements help us gather information about the available options allowing us to recognize them and retrieve corresponding value representations (Schomaker, Walper, Wittmann, \& Einhauser, 2017).

Studies have shown that we typically dwell longer on an object of our preference and choice (e.g. Gidlöf et al., 2017; Jantathai, Danner, Joechl, \& Dürrschmid, 2013; Krajbich et al., 2010; Schotter et al., 2010; Shimojo, Simion, Shimojo, \& Scheier, 2003). Several studies have suggested that the relationship between attentional allocation and choice can be causal: When viewing duration is experimentally manipulated, value-based choice behavior is biased towards the items that were shown longer (Armel, Beaumel, \& Rangel, 2008). Zhang, Wedel, and Pieters (2009) showed the mediating effect of attention on sales. Similar findings have been obtained with more complex decisions such as moral choices (Pärnamets et al., 2015). Of course, consumer choices are also driven by the relative utility or value of the choice alternatives (e.g. Meißner, Musalem, \& Huber, 2016). So, as a basic model of choice, we need to include both attention and value. In addition, attention in and of itself may affect the value of an alternative (Lim, O'Doherty, \& Rangel, 2011).

Viewing behavior (attention) thus plays a crucial role in the valuebased comparator process underlying decision-making (Krajbich \& Rangel, 2011). According to Awh, Belopolsky, and Theeuwes (2012), attention can be driven by top-down factors (e.g. a person's goals), bottom-up factors (distinctiveness of a stimulus relative to its surroundings), or other factors such as selection history, e.g. the mere exposure effect (Zajonc, 1968). For more discussion of this topic see Awh et al. (2012); Orquin and Mueller Loose (2013); Orquin, Perkovic, and Grunert (2018), and Theeuwes (2010). Any factor that may influence eye movements, and thereby attention, can potentially influence choice behavior (e.g. Krajbich \& Rangel, 2011). Hence, bottom-up and top-down factors can possibly directly impact choice or can indirectly impact choice via attention.

Bottom-up factors influencing attention include stimulus features

\footnotetext{
We would like to thank the reviewers of the Journal of Business Research and especially Professor Jacob Orquin \& Professor Michel Wedel for their help and constructive feedback.

* Corresponding author at: Wassenaarseweg 52, 2333 AK Leiden, the Netherlands.

E-mail address: mvriens@uwlax.edu (M. Vriens).
} 
such as position, surface size, contrast, color, brightness, orientation, position, size, symmetry, etc. (e.g. Itti \& Koch, 2000; Itti, Koch, \& Niebur, 1998; Janiszewski, Kuo, \& Tavassoli, 2013; Kootstra, de Boer, \& Schomaker, 2011; Milosavljevic, Navalpakkam, Koch, \& Rangel, 2012; Orquin \& Mueller Loose, 2013; Pieters, Wedel, \& Batra, 2010; Schomaker, Rau, Einhäuser, \& Wittmann, 2017; Theeuwes, 1991, 1992). These bottom-up factors determine the visual salience of an object with respect to its background or other nearby objects. A red apple, for example, will stand out amongst green apples, but not so much amongst other red apples. Visual salience can impact attention (e.g. Awh et al., 2012; Theeuwes, 1992). Several studies have shown that visual salience can directly, or indirectly through its effects on attention, influence decision-making, biasing choice behavior towards more visually salient options (e.g. Chandon, Hutchinson, Bradlow, \& Young, 2009; Lohse, 1997; Milosavljevic et al., 2012; Towal et al., 2013; van der Laan, Hooge, de Ridder, Viergever, \& Smeets, 2015). The findings in the laboratory have been shown to be generalizable to more realistic settings, such as the supermarket (Gidlöf et al., 2017; Zhang et al., 2009).

Visual saliency can be estimated by computational models of visual attention (Itti et al., 1998; Itti \& Koch, 2001; Pieters et al., 2010; van der Lans, Wedel, \& Pieters, 2011), and can be manipulated by changing stimulus features, including colors (e.g. saturation), orientation, size, contrast, or complexity of stimuli relative to the background (Itti et al., 1998; Itti \& Koch, 2000; Pieters et al., 2010). Computational visual salience can affect choice behavior (e.g. Krajbich et al., 2010; Krajbich \& Rangel, 2011; Milosavljevic et al., 2012; Towal et al., 2013). For fast responses and with increasing cognitive load, visual saliency can predict choice better than value, particularly when no strong preferences exist (Milosavljevic et al., 2012). Drift-diffusion models including both value and visual salience are better able to explain and predict viewing and choice behavior than models including only one of these factors (Towal et al., 2013).

Top-down factors include an individual's goals, mood, emotion, and task instructions (e.g. Awh et al., 2012; Öhman, Flykt, \& Esteves, 2001; Pieters \& Wedel, 2007; Rayner, Miller, \& Rotello, 2008; Yarbus, 1967). A top-down attentional set, an attentional bias that promotes the perceptual selection of certain physical properties, may help you direct attention to relevant features. For example, when looking for your favorite brand of shampoo in the supermarket, you may effectively direct your attention to items that match your image of the product or your associations with the brand. Top-down attention can also be guided by externally presented cues, such as arrows, that are aimed to direct attention to a certain location in space (Posner, 1980); for a review discussing different types cue types see Chica, Martín-Arévalo, Botta, and Lupiáñez (2014).

Although, external cues could be used in marketing strategies - for example in shop displays - to direct attention to a new product, the role of externally guided top-down attention in value-based decisionmaking remains under-researched and will be the topic of the current study.

Summarizing, top-down and bottom-up factors may have a direct effect on choice or an indirect effect via attention (e.g. Milosavljevic et al., 2012; Peschel et al., 2019; Towal et al., 2013; Van der Laan et al., 2015).

Many studies investigating the role of attention in decision-making do not explicitly manipulate visual attention. Instead they vary exposure durations to the different options, for example, by showing some items longer than others (e.g. Armel et al., 2008), manipulating the timing of the decision (e.g. Pärnamets et al., 2015), manipulating the first fixation (e.g. van der Laan et al., 2015), or taking differences in visual salience into account (e.g. Chen, Mihalas, Niebur, \& Stuphorn, 2013; Milosavljevic et al., 2012). It currently remains unclear whether similar findings would be obtained if exposure durations between the options were matched, while also accounting for visual salience and top-down attention. Visual attention can effectively be manipulated in a top-down way by using cues (Posner, 1980), such as arrows pointing to the left or right. In many consumer settings, colorful and visually salient products might all compete for attention. In such a situation it becomes difficult to manipulate visual product features in such a way that they stand out from the background. Especially in such cluttered and crowded environments it could be more effective to make consumers shift attention in another way.

Our paper investigates whether a top-down attentional bias as induced by external visual cues, such as arrows pointing to the left or the right, can influence attention and possibly can impact directly or indirectly consumer choice. We present the results of two experiments. Both experiments had two phases. In the first phase of the experiment, participants rated a series of food items on their desirability, by indicating how much they would like to eat each item after the experiment (using a slider from "not at all" to "very much"). In the second phase, participants chose between two food snacks (both with similar ratings). The items were preceded by a directional (left or right) or neutral (left and right) arrow. We expect that directional cues would result in top-down shifts of attention, biasing decision-making processes. We model attention as a function of value, salience, and type of cue (directional versus neutral), and we model consumer choices as a function of value, salience, fixation count (measured via eye tracking as a measure of attention), and type of cue.

\section{Experiment 1}

\subsection{Participants}

31 students from a senior marketing class at the University of Wisconsin-La Crosse, USA, participated in this study. Exclusion criteria were a history of mental illness (also including eating disorders), neurological or psychiatric illness, usage of psychotropic medication in the last three months, and age $<18$ or $>40$ years. When required, eyesight was corrected to normal by contact lenses. All participants gave written informed consent. The study was approved by the Institutional Review Board for the Protection of Human Subjects at the University of Wisconsin, La Crosse. The exclusion criteria described above led to the exclusion of two participants. Data from six additional participants was excluded because of a technical issue during eye tracking (communication between the experimental PC and eye tracker was not stable; i.e., for the eye tracker data, messages regarding condition information were missing for one or more trials, making it impossible to retrace which stimulus was presented when). Data is reported for the remaining set of 23 participants (age range $=20-23 ; \mathrm{M}=21.1$, $\mathrm{SD}=0.8 ; 1$ left-handed). All included participants reported to be in somewhat good to excellent health and reported they regularly snacked. In return for participation, participants were given course credit and a $\$ 5$ Starbucks card.

\subsection{Materials and measures}

Photographs of 12 savory and 22 sweet supermarket snacks acted as stimuli in the current study. All food items and their corresponding desirability ratings are shown in Fig. 1, sorted from low to high mean (the full set of stimuli and stimuli pairs can be found at: https://osf.io/ yeqdw/). For each item the mean, standard deviation, minimum, and maximum desirability rating is shown.

During the choice phase, two snacks were presented simultaneously, and relative visual salience of each item (cued/un-cued) was estimated using the Saliency Toolbox (Walther \& Koch, 2006). This toolbox can be used to calculate saliency maps, based on a bottom-up model of attention to proto-objects, i.e. floating bits of visual information that can be bound into a stable object by focused attention. The saliency maps are based on low-level features, including stimulus intensity, color, and orientation, and a winner-take-all neural network is used to identify the most salient locations (for details regarding the model used, see 


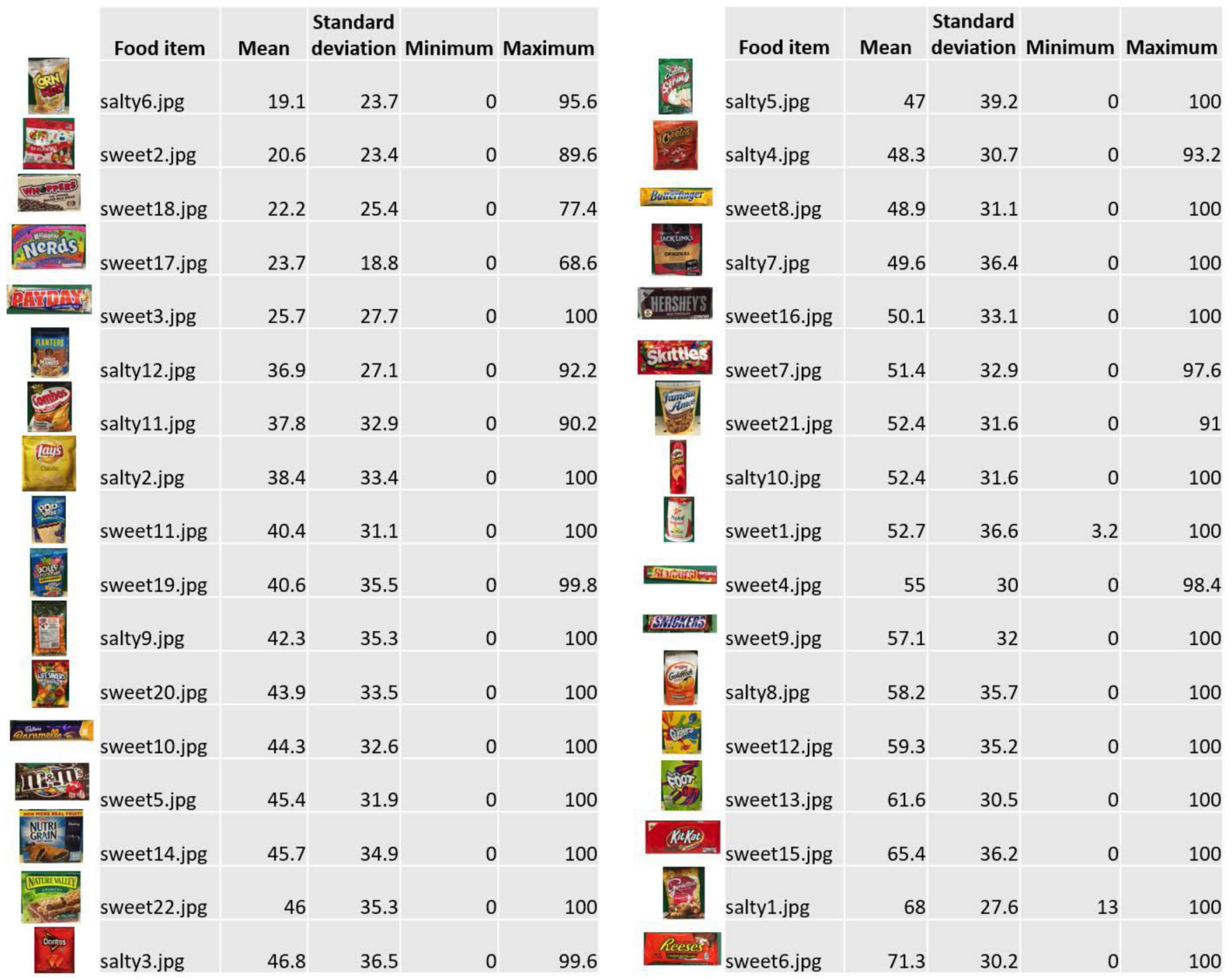

Fig. 1. Overview of stimuli used in Experiments 1 and 2.

Walther \& Koch, 2006). Saliency maps were calculated for each possible combination of items, and peak salience for each item for each combination was determined (based on influential models of attention: Itti \& Koch, 2000; Itti et al., 1998).

\subsection{Apparatus}

The stimuli were presented on a desktop PC (3.6-GHz, 64-bit, 16-GB RAM, and a 24-inch 75- $\mathrm{Hz}$ monitor). The experiment was programmed using Open-Sesame version 3.1 (Mathot, Schreij, \& Theeuwes, 2012). Eye tracking data were collected using the Tobii Pro X20-30 eye tracker at a sampling rate of $30 \mathrm{~Hz}$ using a binocular tracking technique. This eye tracker has $0.4^{\circ}$ accuracy and $0.32^{\circ}$ precision. Open-Sesame and the Tobii eye tracker were linked using the Tobii Software Development Kit.

\subsection{Experimental task}

The main experimental task consisted of a rating and a choice phase. In the ratings phase, participants were first asked to indicate how much they would like to eat the shown item after the experiment. During the rating phase, participants rated each of the snacks on a 1000-point scale (ranging from 0.0 to 100.0 using a slider); participants were not aware the scale was divided in 1000 points, (it was shown as a continuous line). They were asked to indicate how much they would like to eat each of 34 snacks. The rating a snack receives is referred to as the desirability rating or value. Each food item was presented for $2000 \mathrm{~ms}$, after which a fixation cross was presented for $500 \mathrm{~ms}$. After fixation presentation, the slider was presented until a response was recorded. After the rating phase all ratings were sorted, and in the subsequent choice phase, items with neighboring rating scores were shown in pairs, resulting in item pairs with similar ratings. The location of the similarly valued items was randomized, such that the higher and lower valued item could occur on the left or right location. All items and item pairs were presented in a random sequence in both the rating and choice phases. A trial during the choice phase started with a $500 \mathrm{~ms}$ central fixation, followed by a cue presented for a duration of $700 \mathrm{~ms}$. There were three types of cues: one pointing to the left, one to the right, and one consisting of two arrows pointing left and right (i.e., a neutral cue). After the cue presentation, two food items were presented, one to the left and one to the right. Participants were instructed to choose their preferred item. To increase the likelihood that attention effects could play out, participants were instructed to choose quickly without much deliberation. The response time limit was set to $1500 \mathrm{~ms}$. A new trial was initiated once a response was given, or when no valid response was given within this time-window. A chosen object was highlighted by a black box, to confirm the participant's choice. The choice phase consisted of 204 trials. Each cue type (left, neutral, right) was presented on 
Table 1

Results multi-level logistic regression ${ }^{a}$ (binary snack choices, Experiment 1).

\begin{tabular}{lrlrr}
\hline & Coefficient & Standard error & z-Value & p-Value \\
\hline Intercept & -0.07 & 0.07 & -0.99 & 0.32 \\
Left cue & 0.11 & 0.10 & 1.03 & 0.31 \\
Right cue & 0.09 & 0.15 & 0.59 & 0.55 \\
Left salience & 0.15 & 0.08 & 1.90 & 0.06 \\
Right salience & -0.11 & 0.06 & -1.80 & 0.07 \\
Left fixation count & 0.22 & 0.08 & 2.56 & 0.01 \\
Right fixation count & -0.59 & 0.09 & -6.17 & $<0.01$ \\
Left value & 1.45 & 0.29 & 4.99 & $<0.01$ \\
Right value & -1.60 & 0.29 & -5.10 & $<0.01$ \\
\hline
\end{tabular}

a The model is based on $N=23$, and a total of 3221 observations. The AIC value for this model is 4072 , the BIC is 4181 .

68 trials.

\subsection{Procedure}

The experiment was conducted in one of the rooms of a commercial analytics firm: The room was quiet and well-lit. Prior to the actual experiment, subjects filled out a short Qualtrics survey, including questions about their current physical and mental health, snack familiarity, and behavior. Upon arrival on the testing day, participants were briefed about the specific experimental procedures and signed informed consent. First, the participants were shown a table where all the snacks used in our study were displayed. Snacks often come in different sizes and flavors and we wanted to make sure all participants recognized the snacks that would be presented during the experiment. Participants were then shown a few additional online questions, including some regarding last food intake, and how they made their choices. Finally, task instructions were given on paper and understanding of the task was verbally checked. If everything was clear, participants could start the experimental task (as described above in the Experimental task section). The entire experimental procedure took about $1 \mathrm{~h}$ per participant.

\subsection{Eye tracking data acquisition and preparation}

During both phases, eye movements were tracked using a $30-\mathrm{Hz}$ Tobii Pro X20-30 eye tracker. At the beginning of the experiment, participants performed a 9-point calibration and validation until eye tracking was satisfactorily accurate. Fixations were labeled as left or right from the middle of the screen. Fixations on the midline were not categorized. For our analyses, we recoded trials with directional cues to fixations towards the cued or un-cued side. Blinks and missing data points were excluded from analyses $(<5 \%$ missing data for all conditions).

\subsection{Choice and attention models}

We estimated two types of models: A choice model and an attention model. Respondents' choices were modeled using a multi-level (full random effects) logistic regression model (e.g. Guo \& Zhao, 2000) and fixation count (attention) was modeled as a multi-level Poisson regression model (e.g. Beaujean \& Morgan, 2016; Hausman, Hall, \& Griliches, 1984). The dependent variable was the choice for the left item: 1 if chosen, 0 if not chosen (same approach as used by Milosavljevic et al. (2012)). As independent variables we used (1) overall desirability, (2) visual salience, (3) the number of fixations and (4) cue type (i.e. left cue, right cue, neutral cue). For overall desirability, salience, and fixations we have values for the left and the right choice option, so each is represented by two variables.

The model input variables were z-scored to have zero mean and unit standard deviation (Schielzeth, 2010), putting all predictors on a standardized scale, while not affecting the shape of the distributions.
The models were computed using the statistical package $\mathrm{R}$ (version lme4 package is 1.21), including the lme4 R package (Bates, Mächler, Bolker, \& Walker, 2015) version 1.1.7.

Models were evaluated using $p$-values. In addition, we attempted a full step-up analysis (Säfken, Rügamer, Kneib, \& Greven, 2018), i.e. looking at all possible sub-sets, but this approach did not work on our models. Instead, we evaluated the full model against the null model (intercept-only) and several alternative simpler models using AIC (Akaike, 1974) and BIC metrics (Schwarz, 1978) criteria. These measures represent an alternative to using $p$-values (e.g. Wagenmakers, 2007) for evaluating and selecting models. Models with lower AIC and BIC values are preferred.

\subsection{Results}

In total there were 204 trials. Due to the time limit, not all subjects were able to make a choice in time on all trials. The average number of trials was 140 .

The average desirability ratings for the food presented together were similar in value, with an average of 48.16 for items presented on the left, and 48.2 for items presented on the right: the difference is not statistically significant ( $p=0.96$ ), and an average of 46.51 for cued, and 46.40 for un-cued items: the difference is not statistically significant $(p=0.93)$. Note, however, that at the trial-level substantial value differences between the options could occur, depending on the individual's ratings (e.g. when a participant only used the extreme ends of the scale, a larger gap could occur). The average response time for cued chosen was $683.7 \mathrm{~ms}$ and for non-cued chosen $701.8 \mathrm{~ms}$ ( $t$-Test $=-2.9$, $p=004)$. The average fixation time for cued chosen was $393 \mathrm{~ms}$ and for non-cued chosen $290.3 \mathrm{~ms}$ (t-value 16.9, $p<0.001$ ).

Participants chose a food item on 70.3 of the trials but failed to respond on $29.7 \%$ of trials. To further investigate the effects of visual salience, attention (fixation count), and overall value (desirability), we estimated a multi-level logistic regression model as outlined above. The results of this analysis are presented in Table 1 .

As expected, value, visual salience, and attention all have significant effects (i.e. $p$-values $<0.10$ ). The cue variables are not significant. Snacks with higher values are chosen more often, more salient snacks were chosen more often, and snacks that received more attention are chosen more often.

To evaluate the model against alternative models we compared this full model with several alternative models including the null model (intercept-only); a model with intercept and value variables; a model including intercept, value, and salience variables; and a model including intercept, value, salience, and fixations. We compared the set of alternative models with the full model using AIC (Akaike, 1974) and BIC (Schwarz, 1978) criteria. All alternative models have higher AIC values than the full model, though alternative model 4 (a model without the cue variables) had a lower BIC value relative to the full model. Full AIC \& BIC results can be provided upon request.

Next, we ran a multi-level Poisson regression model (using fixation count as the dependent variable). Table 2 shows the results.

Table 2

Results multi-level Poisson regression ${ }^{\mathrm{a}}$ (fixation count, Experiment 1).

\begin{tabular}{lrlrr}
\hline & Coefficient & Standard error & z-Value & p-Value \\
\hline Intercept & 5.90 & 0.06 & 90.76 & $<0.01$ \\
Left cue & 0.18 & 0.03 & 5.65 & $<0.01$ \\
Right cue & -0.41 & 0.11 & -3.56 & $<0.01$ \\
Left salience & 0.01 & 0.01 & 1.70 & 0.09 \\
Right salience & 0.01 & 0.01 & 0.84 & 0.40 \\
Left value & -0.02 & 0.08 & -0.28 & 0.80 \\
Right value & 0.00 & 0.08 & 0.08 & 0.93
\end{tabular}

a The model is based on $\mathrm{N}=23$, and a total of 3221 observations. The AIC value for this model is 343,435 , the BIC is 343,520 . 
The results show that cues have a significant impact on attention. Although cues did not have a direct effect on choice, they do have an indirect effect on choice because they affect attention, and attention significantly affects choice. To test the significance of the indirect effect of cue on choice we applied a mediation analysis using the bootstrap approach proposed by Imai, Keele, and Tingly (2010), using their corresponding R-package (Imai, Keele, Tingly, \& Yamamoto, 2019). This yielded a statistically significant average causal mediation effect of 0.07 , with a lower confidence interval value of 0.05 and an upper confidence value of $0.10(p<0.001)$. We found a direct effect of 0.05 (non-significant) and a total effect of 0.12 with a lower confidence interval value of 0.05 and an upper confidence value of $0.19(p<0.01)$. The odds ratios of indirect, direct and total effect are 1.07, 1.04 and 1.13. Salience, as one would expect, also had a significant effect on choice. The value variables parameters were non-significant.

To evaluate the model against alternative models we compared this full model with several alternative models, using AIC and BIC metrics. The alternative models included the null model (intercept-only); a model with intercept and value variables, and a model including intercept, value, and salience variables. All alternative models have higher AIC and BIC values than the full model.

\section{Experiment 2}

In Experiment 1 we found that cues affected attention and indirectly influenced choices. In contrast to the traditional Posner cueing task, our cue was task irrelevant. The question therefore remains why the cues worked. Potentially participants believed that the cues were relevant, even though we did not instruct them on the cues and their use. Another possibility is that the cueing effect occurs automatically, that is, without conscious attentional deployment.

To further investigate these possibilities, we performed the same experiment again, but varied the instructions between conditions. We used three conditions: a neutral condition, a task relevant condition and a task irrelevant condition. To replicate the first experiment, we included a neutral condition, in which participants were not briefed about the cues. In a cue irrelevant condition, we instructed them that the cues were not relevant and that they should try to ignore them. Finally, in a third cue relevant condition we instructed them that the cues could help them shift their attention and make a choice. If the cues would have an automatic effect on attention, the instructions should not influence viewing and decision-making behavior. If the attention effect on choice only occurs when people voluntarily shift attention to comply with task demands, we would expect to find effects of attention in the relevant condition, while in the neutral condition the attention effect would be smaller, and in the irrelevant condition there would be no effect.

Previous studies have suggested that the effects of attention are transient and hence we included a time-limit in Experiment 1. To check whether we could still obtain effects of attention when response time is not limited, we removed the response time-limit in all three conditions of Experiment 2. Methods were almost the same as in Experiment 1. There were only two differences. The response time limit during the choice phase was removed, allowing participants to fully consider both options, and we gave the participants one of three different instructions regarding the cues before the experiment. We added an open-ended question at the end of the experiment to check what choice strategy participants used to be able to differentiate between cue-based and other strategies.

\subsection{Participants}

Participants of Experiment 2 were students from a senior marketing class at the University of Wisconsin-La Crosse, USA. The same selection and exclusion criteria were used as in Experiment 1. All participants gave written informed consent. The study was approved by the Institutional Review Board for the Protection of Human Subjects
University of Wisconsin, La Crosse. In total 87 participants performed Experiment 2, of which 43 were included in the analyses (age range $=18-24 ; M=20.8$, SD 1.1 years; 6 left-handed, 1 ambidextrous).

In the cue neutral condition, we had 28 participants but data of five participants was excluded because of our exclusion criteria, and an additional four were excluded due to technical issues during eye tracking (due to the same issue as described for Experiment 1). Data is reported for the remaining 19 participants. In the cue irrelevant condition, we had 29 participants. Five participants were excluded because of exclusion criteria, and eight because of technical issues during eye tracking. For the cue irrelevant condition, data is reported for the remaining 16 participants. Finally, 30 participants did the cue relevant condition. Three of these did not meet the inclusion criteria, and 19 were excluded because of technical difficulties during eye tracking. Data is reported for the remaining 8 participants. All included participants reported to be in somewhat good to excellent health and reported they regularly snacked. In return for participation, participants were given course credit and a $\$ 5$ Starbucks card.

\subsection{Results}

The average desirability ratings for the food presented together were similar in value, with an average of 48.48 for items presented on the left, and 48.45 for items presented on the right: the difference is not statistically significant $(p=0.96)$, and an average of 48.5 for cued, and 48.4 for un-cued items: the difference is not statistically significant $(p=0.97)$. Note, however, that on the trial-level substantial value differences between the options could occur, depending on the individual's ratings (e.g. when a participant only used the extreme ends of the scale, a larger gap could occur). The average response time for cued trials was $1037 \mathrm{~ms}$ and for non-cued trials $1059 \mathrm{~ms}$ (t-value $=-1.44$, $p=0.14$ ). The average fixation time for cued chosen was $553 \mathrm{~ms}$ and for non-cued chosen $485 \mathrm{~ms}$ (t-value 9.3, $p<0.001$ ).

To further investigate the effects of visual salience, attention (fixation count), and overall value (desirability) we estimated a multi-level logistic regression model (modeling approach identical to that of Experiment 1). The results of this analysis are presented in Table 3.

As expected, value and attention (fixation count) are both statistically significant. In this case, salience is not, and the cue variables are also not significant. Snacks with higher values are chosen more often and snacks that received more attention are chosen more often.

Several alternative models were evaluated. All alternative models have higher AIC values than the full model, the alternative model without the cue variables had a lower BIC value relative to the full model (see Experiment 1).

Next, we ran a multi-level Poisson regression model (using fixation count as dependent variable). Table 4 shows the results.

The results show that cues have a significant impact on attention. Although cues did not have a direct effect on choice, they do have an

Table 3

Results multi-level logistic regression ${ }^{\text {a }}$ (binary snack choices, Experiment 2).

\begin{tabular}{lrlrr}
\hline & Coefficient & Standard error & z-Value & p-Value \\
\hline Intercept & -0.04 & 0.06 & -0.74 & 0.46 \\
Left cue & -0.06 & 0.07 & -0.95 & 0.34 \\
Right cue & 0.06 & 0.07 & 0.92 & 0.35 \\
Left salience & 0.06 & 0.05 & 1.11 & 0.27 \\
Right salience & -0.07 & 0.05 & -1.28 & 0.20 \\
Left fixation count & 0.33 & 0.09 & 3.83 & $<0.01$ \\
Right fixation count & -0.52 & 0.11 & -4.62 & $<0.01$ \\
Left value & 1.82 & 0.81 & 2.25 & 0.02 \\
Right value & -1.82 & 0.82 & -2.21 & 0.03
\end{tabular}

a The model is based on $N=43$, and a total of 8584 observations. The AIC value of this model is 10,941 , the BIC value is 11,068 . 
Table 4

Results multi-level Poisson regression ${ }^{a}$ (fixation count, Experiment 2).

\begin{tabular}{lrlrr}
\hline & Coefficient & Standard error & z-Value & p-Value \\
\hline Intercept & 6.28 & 0.04 & 162.91 & $<0.01$ \\
Left cue & 0.07 & 0.02 & 3.31 & $<0.01$ \\
Right cue & -0.16 & 0.02 & -6.36 & $<0.01$ \\
Left salience & -0.01 & 0.01 & -1.36 & 0.17 \\
Right salience & -0.02 & 0.01 & -0.17 & 0.09 \\
Left value & -0.03 & 0.06 & -0.41 & 0.70 \\
Right value & -0.01 & 0.06 & -0.08 & 0.93 \\
\hline
\end{tabular}

a The model is based on $\mathrm{N}=43$, and a total of 8584 observation. The AIC value for this model is $1,478,911$, the BIC is $1,479,010$.

indirect effect on choice because they affect attention, and attention significantly affects choice. We again tested the average mediation effect using the bootstrap approach by Imai et al. (2010), and found a statistically significant mediation effect of 0.01 , with a lower confidence interval value of 0.0007 and an upper confidence interval value of $0.02(p=0.04)$. We found a direct effect of 0.005 (non-significant) and a total effect of 0.015 (non-significant). The odds ratios of indirect, direct and total effect are 1.01, 1, and 1.01. Salience, as one would expect, also had a significant effect on attention. The value variables parameters were non-significant. We also evaluated the full model against several alternative, simpler models, identical to Experiment 1, and again the AIC and BIC values were lowest for the full model.

In Experiment 2 we used three different types of cue instructions, i.e.: no instruction/neutral condition (this replicated Experiment 1 except without the time constraint), an instruction that the cues are irrelevant (irrelevant condition) and they should be ignored, and an instruction that the cues are relevant (relevant condition) and should be used. We estimated the full count models for each of these three conditions to investigate if the effect of cue changed. We only found minor differences. Both in the neutral and relevant condition, the cue variables were statistically significant. Even in the irrelevant condition, one of the cue variables had a statistically significant effect.

\subsection{Strategy}

From all 90 participants that we tested in Experiment 2, only three participants mentioned having been influenced by the cues during a self-report questionnaire at the end of the experiment. One participant indicated that the cues influenced their first fixation, but not choice behavior. Another one believed that the cues affected their first fixation and thereby affected their choice. The third believed the cues affected their choices only towards the end of the task. Note, that most participants did not mention the cues at all, and instead mentioned having used a value-driven strategy.

\section{General discussion}

In the current study we present the results of two experiments aimed to investigate whether cues can effectively affect top-down visual attention to influence choices in a binary decision-making task. Top-down attention was manipulated by directional (left or right) cues, and nondirectional cues were presented in a control condition. After the cue, participants were shown two similarly valued food items, simultaneously, one on the left side of the screen, the other on the right side. The task was to choose their preferred item. In contrast with previous work, both choice options were presented for the same duration (Armel et al., 2008; Krajbich et al., 2010). In Experiment 1 there was a timeconstraint to make the choices, in Experiment 2 there was not. Experiment 2 also included different instructions for the subjects on how to possibly use the cues in making their decisions (namely: no instructions on how to use the clues, instructions to ignore them, and instructions to use them).
The effects of attention on choice behavior are typically observed for speeded responses (e.g., Ratcliff, Smith, Brown, \& McKoon, 2016) and short stimulus presentations (Milosavljevic et al., 2012). To allow for effects of attention to occur, participants were asked to give a speeded response, and the response window given per choice was limited to $1500 \mathrm{~ms}$ in Experiment 1. Probably due to these time constraints, participants failed to respond on a substantial number of trials in this Experiment 1. The results showed that attention (measured via fixation count) had a significant effect on snack choices as did overall value of the snacks. This is consistent with several previous studies (Armel et al., 2008; Janiszewski et al., 2013; Krajbich et al., 2010 and Schotter et al., 2010). Cueing had a significant effect on attention and hence had an indirect effect on choice, confirmed via a mediation analysis. This, to our knowledge is a new result.

To test whether the results from Experiment 1 were due to the time constraint imposed we set up a second experiment where we removed the response time limit. ${ }^{1}$ Irrespective of time constraints we observed similar choice and attention (fixation count) results. In both experiments, the effects of the task irrelevant cues did not directly impact choices but had a significant effect on attention, and an indirect effect on choices. Most participants did not mention that the cues affected their choices; the majority did not even mention the cues whatsoever when describing what affected their choices in a final self-report questionnaire. This suggests that the cues influenced participants' gaze and choices, even though they were not aware of the cues influencing their behavior. In the main analyses (Experiments 1 and 2) we found that value affected choice behavior.

Interestingly, without a response time limit in Experiment 2, we replicated the main findings of Experiment 1. This is in contrast with previous studies that have shown that the effects of attention on choice are influenced by time pressure (Milosavljevic et al., 2012; Orquin \& Holmqvist, 2018; Reutskaja, Nagel, Camerer, \& Rangel, 2011; Towal et al., 2013; van der Laan et al., 2015). A reason for this discrepancy may be that the effects of top-down attention are less dependent on time pressure than bottom-up driven effects may be. In our experiment, participants were directing (either consciously or not) their attention to the cued snack option, while in the previous studies attention was typically drawn by salient stimuli or exogenous cues.

To take salience into account in the current study, we used an estimate of the relative salience of the items. Our choice model (Table 1) for Experiment 1 showed that salient items had a higher chance of being chosen than non-salient cued items, but not so in Experiment 2. This is in line with several previous studies that have found that visual salience can influence choice behavior (Chen et al., 2013; Gidlöf et al., 2017; Milosavljevic et al., 2012; Schomaker \& Wittmann, 2017). Maybe due to the relaxation of the time constraint salience failed to have a direct effect on choice in Experiment 2. Some have found that effects of salience are transient (e.g. Donk \& Van Zoest, 2008), some found a salience effect to only occur for the first eye movement and within the first few hundred milliseconds after stimulus presentation, and under specific task conditions (Donk \& Soesman, 2010; Li, Zhang, \& Fine, 2013; Siebold, van Zoest, \& Donk, 2011), while others have found salience to endure beyond the first fixation (e.g. Silvis \& Donk, 2014). When no time constraint exists - as in Experiment 2 - the effects of visual salience may become less pronounced (Stoll, Thrun, Nuthmann, \& Einhauser, 2015), or decrease less quickly as suggested by Donk and Van Zoest (2008) and Orquin and Lagerkvist (2015). Note, salience did have a significant effect on attention in both experiments and hence indirectly impacted choice. Visual salience biasing value-based decision making through its effects on attention could occur in several ways. First, visual salience could increase the speed of attentional selection, i.e., the processing onset (e.g. Chen et al., 2013; Töllner, Zehetleitner, Gramann, \& Müller, 2011). Second, it could generally increase the

\footnotetext{
${ }^{1}$ We thank one of the reviewers for pointing this out
} 
processing speed. One study using stochastic accumulator models showed that both models including effects on onset and speed could explain the effects of visual salience on value-based decisions (Chen et al., 2013). Drift-diffusion models successfully explain the effects of visual salience on decision-making and response times in simple choice tasks (Krajbich \& Rangel, 2011). These models suggest that the decision threshold can be dynamically adapted to fit ongoing goals and make a trade-off between the cost of accumulating more evidence against the cost of the time required to come to a decision (Bogacz, Brown, Moehlis, Holmes, \& Cohen, 2006). Drift-diffusion models incorporating visual salience have shown that models including both value and visual salience explained and predicted viewing and choice behavior better than models that included only one of these factors (Towal et al., 2013).

The task that we used was inspired by the traditional Posner cueing task. In contrast with a traditional Posner cueing task, the cues in our experiment were task irrelevant. They could even guide attention away from a preferred option. Nevertheless, they were effective in influencing top-down attention. These findings were robust: Irrespective of task demands, the cues successfully influenced attention and choice behavior. One reason for this could be that we are so conditioned to use cues, like arrows, in everyday life, that we do not need specific task instructions to do so. Our findings suggest that even irrelevant cues can be effective in guiding attention and influencing choices.

\subsection{Implications}

Attention can play a role in how advertisements and promotions are processed and how they impact consumer choices (e.g. standing in front of a supermarket shelf and deciding what brand to buy (for a review about the role of eye movements in marketing, see Wedel \& Pieters, 2008)). There is tremendous competition for limited attentional resources. Consumers are bombarded with ads and there is strong competition, both at the across-brand and within-brand levels. Both similarity across choice items, and he sheer number of competing alternatives can make the choice hard (e.g. Chernev, Böckenholt, \& Goodman, 2015; Cooper-Martin, 1993; Haynes, 2009). When consumers are strapped for time, they may be even more susceptible to cues. Our findings have several implications. Using cues to attract attention to the brand you would like to promote can be a profitable tactic, as it could increase the chance that a consumer will decide to buy it. Furthermore, as attentional allocation has been linked to successful memory encoding (Muzzio, Kentros, \& Kandel, 2009), cues can help increase the memorability of products, increasing chances the product will be remembered on the next occasion, and thereby increasing chances it may be considered.

\subsection{Limitations and further research}

Past research using eye tracking data to understand choice processes has used a variety of models, of which drift diffusion models (DDMs) are probably most frequently used. DDMs are a family of accumulator models, and have been successful at explaining decision-making behavior and response times in simple tasks, including binary and trinary choices (Krajbich \& Rangel, 2011). The idea behind these models is that information about options is accumulated over time. Once the evidence to favor one option exceeds a certain threshold, a decision is made. The threshold may be dynamically adapted to fit ongoing goals, and to optimize the cost of accumulating more evidence against the cost of the time required to come to a decision (Bogacz et al., 2006). Evidence accumulation, a key feature of this class of models, can be identified in neurological brain activity (Basten, Biele, Heekeren, \& Fiebach, 2010; van Vugt, Beulen, \& Taatgen, 2016). Some DDMs explicitly model the decision-making process over time and allow adjustment to the relative values of the choice items for each fixation. In this study, we did not analyze fixation patterns over time within stimuli, but rather used fixation count to investigate the effectiveness of our main experimental manipulation: the effects of top-down attention on choices. Our experimental paradigm, however, offers a wide range of possibilities for modeling the choice process of within-stimulus fixations over time. Such analyses can be performed using traditional hidden- or semiMarkov modeling (Coutrot, Hsiao, \& Chan, 2018), but also using stateof-the-art deep neural networks (LSTM) for behavioral predictions (Cornia, Baraldi, Serra, \& Cucchiara, 2016). A recent comparison between a DDM and a generalized linear logit model showed that the DDM resulted in better predictive accuracy (Clithero, 2018). However, the differences were small, especially in the case where the choice items were close in initial value. We are not aware of studies that have defined a DDM that included a top-down attention component, which would also be an interesting venue for future research.

\subsection{Conclusion}

Previous studies have already shown that bottom-up attention (e.g. Milosavljevic et al., 2012; Towal et al., 2013) and viewing behavior (Krajbich et al., 2010; Krajbich \& Rangel, 2011) can bias choice behavior, but so far, no studies investigated the role of top-down attention in decision-making. Taken together, our findings suggest that task-irrelevant cues can successfully affect top-down attention and influence choice behavior accordingly. Interestingly, the effects of the cues occurred above and beyond the effects of visual salience and value and occur irrespective of task demands. The arrows were effective cues that made participants involuntarily shift their attention towards the cued location, influencing their choice behavior accordingly. The use of external cues could therefore prove to be an effective way to direct customers' attention and influence purchasing decisions in stimulus-rich shop settings.

\section{Acknowledgments}

We thank M. Groom and A. Baldowin for assistance with data collection and Prof. L.R.B. Schomaker for useful comments. We thank the reviewers of JBR, and especially Professors Michel Wedel and Jacob Orquin for their helpful and constructive feedback.

\section{References}

Akaike, H. (1974). A new look at the statistical model identification. IEEE Transactions on Automatic Control, 19(6), 716-723. https://doi.org/10.1109/TAC.1974.1100705 (MR 0423716).

Armel, K. C., Beaumel, A., \& Rangel, A. (2008). Biasing simple choices by manipulating relative visual attention. Judgment and Decision making, 3(5), 396-403.

Awh, E., Belopolsky, A. V., \& Theeuwes, J. (2012). Top-down versus bottom-up attentional control: A failed theoretical dichotomy. Trends in Cognitive Sciences, 16(8), $437-443$.

Basten, U., Biele, G., Heekeren, H. R., \& Fiebach, C. J. (2010). How the brain integrates costs and benefits during decision making. Proceedings of the National Academy of Sciences of the United States of America, 107(50), 21767-21772.

Bates, D., Mächler, M., Bolker, B. M., \& Walker, S. C. (2015). Fitting mixed linear models. Journal of Statistical Software, 67(1), 1-48.

Beaujean, A. A., \& Morgan, J. B. (2016). Tutorial on using regression models with count outcomes using R. Practical Assessment, Research and Evaluation, 21(2), 1-19.

Bogacz, R., Brown, E., Moehlis, J., Holmes, P., \& Cohen, J. D. (2006). The physics of optimal decision making: A formal analysis of models of performance in two-alternative forced-choice tasks. Psychological Review, 113(4), 700-765.

Chandon, P., Hutchinson, J. W., Bradlow, E. T., \& Young, S. H. (2009). Does in-store marketing work? Effects of the number and position of shelf facings on brand attention and evaluation at the point of purchase. Journal of Marketing, 73(6), 1-17.

Chen, X. M., Mihalas, S., Niebur, E., \& Stuphorn, V. (2013). Mechanisms underlying the influence of saliency on value-based decisions. Journal of Vision, 13(12), 18.

Chernev, A., Böckenholt, U., \& Goodman, J. (2015). Choice overload: A conceptual review and meta-analysis. Journal of Consumer Psychology, 25(2), 333-358.

Chica, A. B., Martín-Arévalo, E., Botta, F., \& Lupiáñez, J. (2014). The spatial orienting paradigm: How to design and interpret spatial attention experiments. Neuroscience and Biobehavioral Reviews, 40, 35-51.

Clithero, J. A. (2018). Improving out-of-sample predictions using response times and a model of the decision process. Journal of Economic Behavior \& Organization, 148, 344-375.

Cooper-Martin, E. (1993). Effects of information format and similarity among alternatives on consumer choice processes. Journal of the Academy of Marketing Science, 21(3), 
239-246.

Cornia, M., Baraldi, L., Serra, G., \& Cucchiara, R. (2016). Predicting human eye fixations via an LSTM-based saliency model. IEEE Transactions on Image Processing, 27(10), 5142-5154.

Coutrot, A., Hsiao, J. H., \& Chan, A. B. (2018). Scanpath modeling and classification with hidden Markov models. Behavior Research Methods, 50(1), 362-379.

Deubel, H., \& Schneider, W. X. (1996). Saccade target selection and object recognition; evidence for a common attentional mechanism. Vision Research, 36(12), 1827-1837.

Donk, M., \& Soesman, L. (2010). Salience is only briefly represented: Evidence from probe-detection performance. Journal of Experimental Psychology: Human Perception and Performance, 36(2), 286-302.

Donk, M., \& Van Zoest, W. (2008). Effects of salience are short-lived. Psychological Science, 19(7), 733-739.

Gidlöf, K., Anikin, A., Lingonblad, M., \& Wallin, A. (2017). Looking is buying. How visual attention and choice are affected by consumer preferences and properties of the supermarket shelf. Appetite, 116, 29-38.

Guo, G., \& Zhao, H. (2000). Multilevel modeling of binary data. Annual Review of Sociology, 26, 441-462.

Hausman, J., Hall, B. H., \& Griliches, Z. (1984). Econometric models for count data with an applicationto the patents-R\&D relationship. Econometrica, 52, 909-938.

Haynes, G. A. (2009). Testing the boundaries of the choice overload phenomenon: The effect of number of options and time pressure on decision difficulty and satisfaction. Psychology \& Marketing, 26(3), 204-212.

Imai, K., Keele, L., \& Tingly, D. (2010). A general approach to causal mediation analysis. Psychological Methods, 15(4), 309-334.

Imai, K., Keele, L., Tingly, D., \& Yamamoto, T. (2019). https://cran.r-project.org/web/ packages/mediation/vignettes/mediation-old.pdf.

Itti, L., \& Koch, C. (2000). A saliency-based search mechanism for overt and covert shifts of visual attention. Vision Research, 40(10-12), 1489-1506.

Itti, L., \& Koch, C. (2001). Computational modelling of visual attention. Nature Reviews Neuroscience, 2(3), 194-203.

Itti, L., Koch, C., \& Niebur, E. (1998). A model of saliency-based visual attention for rapid scene analysis. IEEE Transactions on Pattern Analysis and Machine Intelligence, 20(11), 1254-1259.

Janiszewski, C., Kuo, A., \& Tavassoli, N. T. (2013). The influence of selective attention and inattention to products on subsequent choice. Journal of Consumer Research, 39(6), 1258-1274.

Jantathai, S., Danner, L., Joechl, M., \& Dürrschmid, K. (2013). Gaze behavior, choice and color of food: Does gazing behavior predict choice? Food Research International, 54, $1621-1626$.

Kootstra, G., de Boer, B., \& Schomaker, L. R. B. (2011). Predicting eye fixations on complex visual stimuli using local symmetry. Cognitive Computation, 3(1), 223-240.

Krajbich, I., Armel, C., \& Rangel, A. (2010). Visual fixations and the computation and comparison of value in simple choice. Nature Neuroscience, 13(10), 1292-1298.

Krajbich, I., \& Rangel, A. (2011). Multialternative drift-diffusion model predicts the relationship between visual fixations and choice in value-based decisions. Proceedings of the National Academy of Sciences of the United States of America, 108(33), $13852-13857$.

Li, H. M., Zhang, H., \& Fine, C. H. (2013). Dynamic business share allocation in a supply chain with competing suppliers. Operations Research, 61(2), 280-297.

Lim, S.-L., O'Doherty, J. P., \& Rangel, A. (2011). The decision value computations in the vmPFC and striatum use a relative value code that is guided by visual attention. The Journal of Neuroscience, 31(37), 13214-13223.

Lohse, G. L. (1997). Consumer eye movement patterns on yellow pages advertising. Journal of Advertising, 26(1), 61-73.

Mathot, S., Schreij, D., \& Theeuwes, J. (2012). OpenSesame: An open-source, graphical experiment builder for the social sciences. Behavior Research Methods, 44(2), 314-324.

Meißner, M., Musalem, A., \& Huber, J. (2016). Eye tracking reveals processes that enable conjoint choices to become increasingly efficient with practice. Journal of Marketing Research, LIII, 1-17.

Milosavljevic, M., Navalpakkam, V., Koch, C., \& Rangel, A. (2012). Relative visual saliency differences induce sizable bias in consumer choice. Journal of Consumer Psychology, 22(1), 67-74.

Muzzio, I. A., Kentros, C., \& Kandel, E. (2009). What is remembered? Role of attention on the encoding and retrieval of hippocampal representations. Journal of Physiology (London), 587(12), 2837-2854.

Öhman, A., Flykt, A., \& Esteves, F. (2001). Emotion drives attention: Detecting the snake in the grass. Journal of Experimental Psychology, 130(3), 466-478.

Orquin, J. L., \& Holmqvist, K. (2018). Threats to the validity of eye-movement research in psychology. Behavior Research Methods, 50(4), 1645-1656.

Orquin, J. L., \& Lagerkvist, C. J. (2015). Effects of salience are both short- and long-lived. Acta Psychologica, 160, 69-76.

Orquin, J. L., \& Mueller Loose, S. (2013). Attention and choice: A review on eye movements in decision making. Acta Psychologica, 144(1), 190-206.

Orquin, J. L., Perkovic, S., \& Grunert, K. G. (2018). Visual biases in decision making. Applied Economic Perspectives and Policy, 40(4), 523-537.

Pärnamets, P., Johansson, P., Hall, L., Balkenius, C., Spivey, M. J., \& Richardson, D. C. (2015). Biasing moral decisions by exploiting the dynamics of eye gaze. Proceedings of the National Academy of Sciences of the United States of America, 112(13), 4170-4175.
Peschel, A. O., Orquin, J. L., \& Mueller Loose, S. (2019). Increasing consumers' attention capture and food choice through bottom-up effects. Appetite, 132, 1-7.

Pieters, R., Warlop, L., \& Wedel, M. (2002). Breaking through the clutter: Benefits of advertisement originality and familiarity for brand attention and memory. Management Science, 48(6), 765-781.

Pieters, R., \& Wedel, M. (2007). Goal control of attention to advertising: The Yarbus implication. Journal of Consumer Research, 34(2), 224-233.

Pieters, R., Wedel, M., \& Batra, R. (2010). The stopping power of advertising: Measures and effects of visual complexity. Journal of Marketing, 74(5), 48-60.

Posner, M. I. (1980). Orienting of attention. Quarterly Journal of Experimental Psychology, 32(1), 3-25.

Ratcliff, R., Smith, P. L., Brown, S. D., \& McKoon, G. (2016). Diffusion decision model: Current issues and history. Trends in Cognitive Sciences, 20(4), 260-281.

Rayner, K., Miller, B., \& Rotello, C. M. (2008). Eye movements when looking at print advertisements: The goal of the viewer matters. Applied Cognitive Psychology, 22, 697-707.

Reutskaja, E., Nagel, R., Camerer, C. F., \& Rangel, A. (2011). Search dynamics in consumer choice under time pressure: An eye-tracking study. American Economic Review, 101(2), 900-926.

Säfken, B., Rügamer, D., Kneib, T., \& Greven, S. (2018). Conditional model selection in mixed-effects models with cAIC4. Electronic Journal of Statistics, 8, 201-225. https:// doi.org/10.1214/14-EJS881.

Schielzeth, H. (2010). Simple means to improve the interpretability of regression coefficients: The interpretation of regression coefficients. Methods in Ecology and Evolution, 1(2), 103-113.

Schomaker, J., Rau, E. M., Einhäuser, W., \& Wittmann, B. C. (2017). Motivational objects in natural scenes (MONS): A database of $>800$ objects. Frontiers in Psychology, 8, 1669.

Schomaker, J., Walper, D., Wittmann, B. C., \& Einhauser, W. (2017). Attention in natural scenes: Affective-motivational factors guide gaze independently of visual salience. Vision Research, 133, 161-175.

Schomaker, J., \& Wittmann, B. C. (2017). Memory performance for everyday motivational and neutral objects is dissociable from attention. Frontiers in Behavioral Neuroscience, 11. https://doi.org/10.5281/zenodo.802156.

Schotter, E. R., Berry, R. W., McKenzie, C. R. M., \& Rayner, K. (2010). Gaze bias: Selective encoding and liking effects. Visual Cognition, 18(8), 1113-1132.

Schwarz, G. E. (1978). Estimating the dimension of a model. Annals of Statistics, 6(2), 461-464. https://doi.org/10.1214/aos/1176344136.

Shimojo, S., Simion, C., Shimojo, E., \& Scheier, C. (2003). Gaze bias both reflects and influences preference. Nature Neuroscience, 6(12), 1317-1322.

Siebold, A., van Zoest, W., \& Donk, M. (2011). Oculomotor evidence for top-down control following the initial saccade. PLoS One, 6(9), e23552.

Silvis, J. D., \& Donk, M. (2014). The effects of saccade-contingent changes on oculomotor capture: Salience is important even beyond the first oculomotor response. Attention, Perception \& Psychophysiology, 76, 1803-1814.

Stoll, J., Thrun, M., Nuthmann, A., \& Einhauser, W. (2015). Overt attention in natural scenes: Objects dominate features. Vision Research, 107, 36-48.

Theeuwes, J. (1991). Cross-dimensional perceptual selectivity. Perception \& Psychophysics, 50(2), 184-193.

Theeuwes, J. (1992). Perceptual selectivity for color and form. Perception \& Psychophysics, 51(6), 599-606.

Theeuwes, J. (2010). Top-down and bottom-up control of visual selection. Acta Psychologica, 135(2), 77-99.

Töllner, Zehetleitner, Gramann, \& Müller (2011). Stimulus saliency modulates pre-attentive processing speed in humans visual cortex. PLoS One, 6(1), 1-8.

Towal, R. B., Mormann, M., \& Koch, C. (2013). Simultaneous modeling of visual saliency and value computation improves predictions of economic choice. Proceedings of the National Academy of Sciences, 110(40), E3858-E3867.

van der Laan, L. N., Hooge, I. T. C., de Ridder, D. T. D., Viergever, M. A., \& Smeets, P. A. M. (2015). Do you like what you see? The role of first fixation and total fixation duration in consumer choice. Food Quality and Preference, 39, 46-55.

van der Lans, R., Wedel, M., \& Pieters, R. (2011). Defining eye-fixation sequences across individuals and tasks: The Binocular-Individual Threshold (BIT) algorithm. Behavior Research Methods, 43(1), 239-257.

van Vugt, M. K., Beulen, M. A., \& Taatgen, N. A. (2016). Is there neural evidence for an evidence accumulation process in memory decisions? Frontiers in Human Neuroscience, 10, 1-13. https://doi.org/10.3389/fnhum.2016.00093 article 93.

Wagenmakers, e.-J. (2007). A practical solution to the pervasive problem of p-values. Psychonomic Bulletin \& Review, 14(5), 779-804.

Walther, D., \& Koch, C. (2006). Modeling attention to salient proto-objects. Neural Networks, 19(9), 1395-1407.

Wedel, M., \& Pieters, R. (2008). A review of eye-tracking research in marketing. Review of marketing research (pp. 123-147). Emerald Group Publishing Limited.

Yarbus, A. L. (1967). Eye movements and vision. New York: Plennum.

Zajonc, R. B. (1968). Attitudinal effects of mere exposure. Journal of Personality and Social Psychology, 9(2), 1-27. https://doi.org/10.1037/h0025848.

Zhang, J., Wedel, M., \& Pieters, R. (2009). Sales effects of attention to feature advertisements: A Bayesian mediation analysis. Journal of Marketing Research, 46(5), $669-681$. 